Fire Ant Polymorphism: The Ergonomics of Brood Production

Author(s): Sanford D. Porter and Walter R. Tschinkel

Source: Behavioral Ecology and Sociobiology, Vol. 16, No. 4 (1985), pp. 323-336

Published by: Springer

Stable URL: http://www.jstor.org/stable/4599785

Accessed: 03-11-2015 20:57 UTC

Your use of the JSTOR archive indicates your acceptance of the Terms \& Conditions of Use, available at http://www.jstor.org/page/ info/about/policies/terms.jsp

JSTOR is a not-for-profit service that helps scholars, researchers, and students discover, use, and build upon a wide range of content in a trusted digital archive. We use information technology and tools to increase productivity and facilitate new forms of scholarship. For more information about JSTOR, please contact support@jstor.org. 


\title{
Fire ant polymorphism: the ergonomics of brood production
}

\author{
Sanford D. Porter and Walter R. Tschinkel \\ Department of Biological Science, Florida State University, Tallahassee, Florida 32306-3050, USA \\ Received March 13, 1984 / Accepted August 31, 1984
}

Summary. Social organization is generally assumed to increase colony efficiency and survival; however, little quantitative information is available to support this assumption. Polymorphism is an important aspect of labor division in colonies of the fire ant, Solenopsis invicta. Our objective was to investigate the effect of fire ant polymorphism on brood production efficiency. We set up standardized polymorphic colonies with a full range of worker sizes and artificial monomorphic colonies that contained only small, medium or large workers respectively. Polymorphic colonies produced brood at about the same rate as colonies composed of only small workers (Fig. 2A). Colonies composed of only medium workers produced about $30 \%$ less brood, and colonies composed of only large workers produced little or no brood at all. This pattern was independent of colony size; however, smaller colonies $(0.75 \mathrm{~g}$, live weight) produced almost twice as much brood per gram of workers as larger colonies $(3.0 \mathrm{~g})$. Additional experiments revealed that the size of workers in the artificial monomorphic colonies affected all stages of brood rearing. Large workers not only inhibited the development of early and late instar larvae (Fig. 4), but also reduced the queen's oviposition rate (Fig. 3). Brood production efficiency on an energetic basis was determined by dividing the grams of brood produced per unit time by the energetic costs expended for the maintenance and production of each worker size class. Worker maintenance costs were estimated from respiration, while production costs were determined from the caloric content of worker tissue divided by their average longevity. Worker respiration per milligram body weight decreased about $40 \%$ as body size increased (Fig. 5). Large workers lived about $50 \%$ longer than small workers (Fig. 6) and contained 9\% more energy per milligram of tissue (Fig. 7). Ener- getic efficiency in polymorphic colonies was approximately $10 \%$ higher than in colonies composed of only small workers (Fig. 9). In other words, when food supplies are limiting, polymorphism may offer a slight advantage in brood production.

\section{Introduction}

Worker size variation is an important component of labor division in colonies of the fire ant Solenopsis invicta Buren. Workers from the same colony may exhibit a 3-fold variation in length and more than a 15 -fold variation in weight (Fig. 1). Small workers are most important in rearing brood but can perform all colony tasks (Mirenda and Vinson 1981; Wilson 1978). The function of large workers is not as clear, but they appear to be more important in mound construction (Wilson 1978), foraging for insect prey (Mirenda and Vinson 1981), transport of sexual brood (O'Neal and Markin 1973), territory defense (Wilson et al. 1971) and perhaps food storage (Howard and Tschinkel 1981).

The degree of worker size variation generally depends on colony size and age. Workers from young colonies are nearly monomorphic; they tend to be small and normally distributed about their mean (Wood and Tschinkel 1981). Workers from older and larger colonies vary considerably in size; they tend to be somewhat larger with frequency distributions strongly skewed toward larger workers (Greenberg et al., in press).

Why are mature fire ant colonies polymorphic? What is the ergonomic advantage (Wilson 1968) of having both large and small workers in the same colony? The inclusion of a range of worker sizes 
may either enhance or reduce the ergonomic efficiency of specific colony tasks (Oster and Wilson 1978), but according to evolutionary theory, the overall effect should be adaptive. Much of sociobiological theory (Wilson 1975) rests on the assumption that social organization increases colony fitness; however, relatively little quantitative information is available to support this assumption (Wilson 1980).

Our objective was to investigate the adaptive value of fire ant polymorphism in relation to brood production. We selected brood production over other colony tasks because it is a key component in colony energetics and labor efficiency. Two major questions will be addressed: (1) How does worker size affect the efficiency of colony brood production? and (2) Are polymorphic colonies more efficient in brood production than monomorphic colonies? Our initial hypothesis was that the inclusion of larger workers in polymorphic colonies would reduce brood rearing efficiency because large workers are more costly to produce and less inclined to tend brood (Mirenda and Vinson 1981).

\section{Materials and methods}

Calculation of brood production efficiency. The best measure of colony fitness is the number of viable sexuals produced over the life of the colony (Wilson 1968); unfortunately, this value is very difficult to measure. Worker brood production was selected as an alternative indicator because it is a central element of colony growth and energy consumption. High rates of worker brood production eventually translate into larger colonies. Larger colonies, in turn, are more capable of producing sexual reproductives (Markin et al. 1973). Furthermore, it seems reasonable to assume that the capacities to produce worker brood and sexual brood are usually positively associated. This study of brood production efficiency was intended as a first step toward determining the adaptive value of fire ant polymorphism; future studies of other colony tasks will be necessary before the overall value of fire ant polymorphism can be estimated.

We determined brood production efficiencies first on a biomass basis and then on an energetic basis. Biomass efficiencies were determined by dividing observed brood production rates by colony biomass. Energetic brood production efficiencies were calculated by dividing brood production rates by the energetic costs of producing and maintaining each size class of ants:

\section{Brood production rate

$\begin{gathered}\text { Energetic brood } \\ \text { production efficiency }\end{gathered}=\frac{}{\text { (maintenance cost }+ \text { production cost })}$

Results of this benefit to cost ratio were given in grams of brood produced (dry weight) per kcal of energy expended. Maintenance costs were determined by measuring respiration rates of each worker size class and then converting oxygen consumption to energy consumption at the rate of $4.8 \mathrm{cal} / \mathrm{ml}$ of oxygen consumed assuming a mixed diet (Elliot and Davison 1975). Production costs were estimated by dividing the pergram caloric content of each worker size class by their average longevity; this prorated or distributed production costs over the entire life of the worker. Prorating production costs was important because it converted them into costs per unit time, which could then be added to maintenance costs.

Our estimates of brood production efficiency were determined with unlimited food supplies. Fire ant colonies may have unlimited food resources when invading previously unoccupied habitat or during seasonal surges in food abundance. Nevertheless, the general assumption that fire ant colonies are foodlimited seems most reasonable because colonies are frequently found in very high densities (Hays et al. 1982) and workers actively defend foraging territories (Wilson et al. 1971). As food resources become increasingly limited, colonies with higher energetic brood production efficiencies should be able to produce more brood per quantity of food energy consumed.

Because our objective was to investigate the effect of polymorphism on brood production efficiency, we attempted to minimize or eliminate the impact of other colony activities on brood production. Effects of mound construction were eliminated because our laboratory colonies contained no soil and could not thermoregulate. Similarly, experimental colonies had no occasion to defend their mounds or their foraging territories. Sexual production in the main experiment and the egg rearing experiment was minimal because of small colony size. Energetic costs of foraging were minimized by feeding experimental colonies ad libitum so that colonies did not need to choose between shunting energy from brood production to worker maintenance or vice versa. We hope these procedures allowed brood production to be estimated relatively independently of energy constraints and the influence of other major colony tasks. However, we were not able to control for the effects of food transfer, storage or digestion. If worker size is important in the distribution and processing of colony food, this effect may have substantially affected our observed rates of brood production.

Sieving and sorting procedures. Workers were sorted into six size classes using USA standard testing sieves (No. 16-35, Fig. 1; Wood and Tschinkel 1981) whose inner walls were coated with fluon (Banks et al. 1981). Brood and sexuals were removed from colonies prior to sieving. Workers were then placed in the top of the stack and allowed to crawl down through the sieves until the mesh size became too small to allow passage. Since the entire sieving process required $4-5 \mathrm{~h}$, a small amount of water was occasionally squirted onto the screens to minimize worker dehydration. After the first half hour, sieves were generally removed from the stack and placed into individual trays to speed sorting. Frequent banging and careful use of a $60 \mathrm{~W}$ light to induce negative phototaxis further encouraged separation.

Sieving was about $90 \%$ effective in sorting workers into appropriate size categories ${ }^{1}$; of the remaining $10 \%$, most were no more than one size too small for the sieve category they selected. Head widths were measured with a wedge micrometer (Porter 1983) to the nearest $0.01 \mathrm{~mm}$. Mean worker dry weights averaged $47 \pm 4 \%$ of wet weight (range $39-55 \%$ ). There was no significant relationship between worker size and percent water content.

For the main experiment and also the egg laying, egg rearing and larval rearing experiments, we set up four treatments of colony composition: polymorphic colonies with a full range of worker sizes and artificial monomorphic or pseudomutant (Wilson 1980) colonies containing only small, medium or large

1 Average head widths $(\mathrm{mm} \pm \mathrm{SD})$ and dry weights $(\mathrm{mg} \pm \mathrm{SD})$ for workers collected on each sieve size were as follows: no. $16-1.35 \pm 0.04,2.21 \pm 0.41$; no. $18-1.19 \pm 0.08,1.56 \pm 0.35$; no. $20-1.01 \pm 0.05,1.02 \pm 0.23$; no. $25-0.86 \pm 0.05$, $0.62 \pm 0.17$; no. $30-0.74 \pm 0.04,0.39 \pm 0.10$; no. $35-$ $0.68 \pm 0.02,0.29 \pm 0.06$ 
workers. Polymorphic colonies contained a standardized ratio (by live weight) of workers collected from all six sieve sizes as follows: no. $16-6 \%$, no. $18-13 \%$, no. $20-9 \%$, no. $25-15 \%$, no. $30-30 \%$ and no. $35-27 \%$. This frequency distribution was chosen to approximate that observed in mature fire ant colonies (Fig. 1). Small-worker colonies contained ants collected on sieve no. 35, medium-worker colonies contained ants collected from sieve no. 25 and large-worker colonies were composed of workers removed from sieve nos. 18 and $16(67 \%$ and $33 \%$ by live weight). With two exceptions, we did not set up artificial monomorphic colonies with either mediumsmall (sieve no. 30) or medium-large (sieve no. 20) workers.

Workers were separated from brood by lightly etherizing a sample and scattering it onto a large sheet of construction paper. As workers revived, they clung to the paper allowing brood to be shaken off (Banks et al. 1981); this procedure was repeated until separation was complete.

Experimental colonies were maintained at $30.0 \pm 0.7^{\circ} \mathrm{C}$ in constant darkness except during maintenance. They were fed ad libitum quantities of cockroaches (Periplaneta) or tenebrionid larvae (Zophobas) and $1 \mathrm{M}$ sucrose soaked into small tissue wads. Colony collection and handling techniques were similar to those described by Banks et al. (1981) and Tschinkel and Howard (1978).

Respirometry. Respiration was measured with modified Scholander (constant pressure) respirometers similar to those described by Wilson (1980) and Davies (1966). Respiration chambers were $20 \times 75 \mathrm{~mm}$ screw-top vials attached horizontally to a compensation chamber partially filled with colored water. A coil of filter paper was placed in the end of each respiration chamber and saturated with $4 \% \mathrm{KOH}$ solution to absorb evolved carbon dioxide. We used a $4 \%$ solution to maintain high humidity in the respiration chambers (Ettershank and Whitford 1973) thus minimizing worker dehydration. Ants were separated from the $\mathrm{KOH}$ coil by a fine mesh screen held in place by $\mathrm{C}$-rings cut from large diameter surgical tubing. Temperature was maintained at $30 \pm 0.5^{\circ} \mathrm{C}$ by immersing the entire respirometer in a covered water bath.

Consumption of oxygen in the respiration chamber caused colored water in the compensation chamber to rise through a thin tube connecting the two chambers. The quantity of oxygen consumed was measured by injecting oxygen into the respiration chamber with a syringe until the colored water was restored to its original level. Respiration chambers were pressurized at the conclusion of each test to insure that all seals had been air-tight.

Longevity. We immobilized newly eclosed callow workers on crushed ice and measured their head widths under an ocular micrometer. Immobilized workers were then marked by tying different colored wire rings between the gaster and the postpetiole (Mirenda and Vinson 1979). Workers with head widths less than $0.8 \mathrm{~mm}$ were marked with $1 \mathrm{mil}(0.025 \mathrm{~mm})$ nichrome wire (Driver-Harris Co., 310 Middlesex St., Harrison, NJ 07029, USA). The ants were immobilized on ice for about 45 min during the sorting and marking procedures.

One day following collection, marked callow ants were added to colonies which contained $1.5 \mathrm{~g}$ (live weight) of dark workers and $0.5 \mathrm{~g}$ of brood. These colonies were placed in fluoned nursery trays containing $150 \mathrm{~mm}$ petri dish nests with dental plaster floors (Banks et al. 1981). A queen was adopted into each colony one week after set-up. Additional nests were added as colonies grew.

Calorimetry. Workers from five colonies were sieved into size classes, killed and dried at $60{ }^{\circ} \mathrm{C}$ for $24 \mathrm{~h}$. Dried workers were then ground with a mortar and pestle and compacted into ap- proximately $0.5 \mathrm{~g}$ pellets. The pellets were burned in a Parr bomb calorimeter using procedures described by Shoemaker et al. (1981). Benzoic acid was used as a standard to calibrate the bomb.

\section{Results}

\section{Worker polymorphism}

Workers from 34 mature fire ant colonies were collected and sieved during the course of our investigations; the number and live weight of workers collected averaged $38,000 \pm 14,000$ and $40 \pm 16 \mathrm{~g}$ respectively. Based on Markin et al. (1973), these colonies were at least 2-3 years old. Our collections usually included a large fraction of the colony population but no attempt was made to collect the entire colony. An effort was made to exclude both young and extremely large colonies.

Size distributions of fire ants collected from these colonies were strongly skewed toward larger workers (Fig. 1). Larger workers contributed substantially more to colony biomass (Fig. 1B) than to colony population (Fig. $1 \mathrm{~A}$ ). Workers in the two largest size classes accounted for $19 \pm 5 \%$ of worker biomass but only $6 \pm 2 \%$ of worker population, while workers in the two smallest size classes accounted for $76 \pm 5 \%$ of worker number but only $53 \pm 7 \%$ of worker biomass. The distribution of worker sizes differed considerably among colonies. For instance, the biomass of workers in the smallest size class (35) ranged from $1-43 \%$ of the total sample weight. Similarly, workers in the two largest size classes $(16,18)$ ranged from $9-32 \%$ of total sample weight.

The relationship between body weight and head width was linear on a log-log plot $(y=$ $0.933 x^{2.98}, R^{2}=93 \%$ ). The coefficient and exponent of the independent variable were very near that expected for isometric growth where volume increases directly according to the cube of dimension. It is also interesting that percent variation along the regression line was nearly constant. In other words, larger workers do not exhibit an increased percentage of weight variation which would be characteristic of a specialized food storage caste (Wilson 1978). At any given head width, dry weight varied nearly $100 \%$, probably due to age-related weight changes (Porter and Jorgensen 1981) and intercolony weight differences.

\section{Brood production}

\section{Main experiment}

Design and procedures. Effects of worker size on brood production rates were studied in a 3-way 

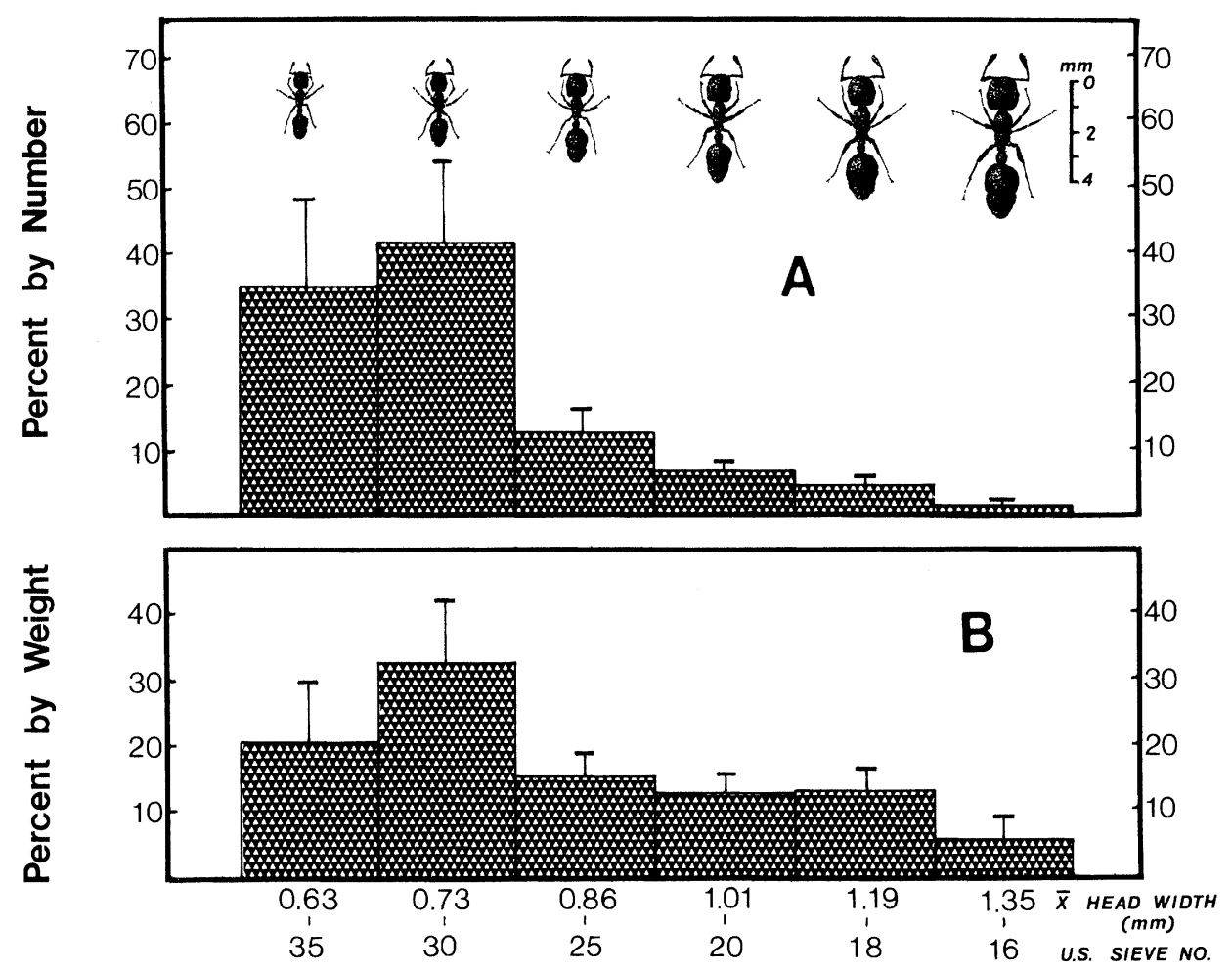

Fig. 1. A Percent distribution of worker population by size class.

B Percent distribution of worker biomass by size class. Data are averages from 34 mature fire ant colonies; standard deviations are indicated for each bar

\section{Worker Size Class}

factorial experiment. Colony composition and colony size were the two experimental factors and source colony was a blocking or random effect factor.

Colony composition had four treatments: polymorphic colonies and monomorphic colonies composed of only small, medium or large workers. Colony size was chosen as a second experimental factor because it is correlated with the degree of polymorphism in field colonies. Our experiment contained two levels: small colonies with $0.75 \mathrm{~g}$ of workers and larger colonies with $3.0 \mathrm{~g}$ of workers. These colony sizes are typical of field colonies which are 4 and 6 month old respectively (Markin et al. 1973). The number of workers in each colony was adjusted so that worker biomass remained constant across colony composition treatments. As a result, colonies of small workers contained about twice as many ants as colonies of medium workers and about six times as many ants as colonies of large workers. Similarly, polymorphic colonies contained about $40 \%$ fewer ants than did smallworker colonies. Biomass was held constant to equalize the standing energy investment in each of the treatment groups.

Workers from different colonies may differ widely in their brood-producing capabilities; to ac- count for this, we used worker source colony as a blocking factor. This was accomplished by sieving a large colony (40-80 g of workers) and then dividing it into eight fragment colonies of the appropriate size and worker composition. Seven source colonies were used, each providing one replicate set of eight experimental colonies. Source colonies one and two were collected and set up in April 1982. Source colonies three and four were set up in May 1982. Source colonies five, six and seven were set up in March, April and May 1983, respectively. Prior to sieving, source colonies were de-queened and held in the laboratory for 5-7 days with their brood.

Mature queens from laboratory stock colonies were randomly adopted into each experimental colony. In one case, the adopted queen was apparently executed, but a second queen was successfully adopted within three days. The experimental colonies were placed in nests containing either two or eight nest chambers $(75 \times 50 \times 3.2 \mathrm{~mm})$ depending on colony size. These chambers were connected by $3 \mathrm{~mm}$ wide tunnels and covered with doublewide microscope slides. Nests were cast from dental plaster (Castone) and contained an internal watering tube used to keep the plaster moist. Plaster nests were placed inside nursery trays ( 20 by 
$20 \mathrm{~cm}$ or 52 by $26 \mathrm{~cm}$ ) which served as foraging arenas. Sides of these trays were coated with fluon to prevent colony escape.

Colonies were fed ad libitum so that food supply would not be a limiting factor. Dead workers were removed at weekly intervals and replaced with live workers from a reservoir so that colony size remained relatively constant throughout the experiment. After 4 weeks, just before the first pupae eclosed, brood were collected and removed from the colonies as previously described. A large fraction of eggs and early instar larvae, however, were not removed from the colonies because they stuck to the workers. (This was not the situation when the source colonies were initially set up because almost all eggs and small larvae had been lost during collection and sorting from the soil.) Experimental colonies were then allowed to produce brood for $2^{1} / 2$ additional weeks before a second and final brood collection.

With the sixth and seventh source colonies, we made two changes in experimental procedure which were designed to facilitate the initiation of brood production. First, experimental colonies were set up with either 1.5 or $0.38 \mathrm{~g}$ (depending on colony size) of third and fourth instar larvae. The resulting pupae were removed after eight days before most of the eggs laid during that period began hatching. The second change was that the eggs and very small larvae which passed through a no. 30 sieve were returned to the colony when larger brood was removed at the end of the first 4 weeks.

Results. Brood production during the first four weeks of the main experiment was generally low and irregular, probably owing to the initial lack of brood in the experimental colonies. Late fourth instar larvae stimulate a queen's egg-laying (Tschinkel, unpublished data) and may also indirectly promote growth of younger larvae through digestion of protein for the colony as a whole (O'Neal and Markin 1973; Petralia and Vinson 1978). Low brood production was especially severe with experimental replicates four and five. Queens in these 16 colonies were observed to lay a large supply of eggs, but only a few larvae resulted and most of those did not develop into pupae. When brood production failed to accelerate after the first month, these colonies were eliminated from the experiment. Four additional colonies were eliminated because their queens died and one $3.0 \mathrm{~g}$ polymorphic colony (replicate six) was excluded because it failed to produce pupae. Missing values were estimated and the results analyzed using
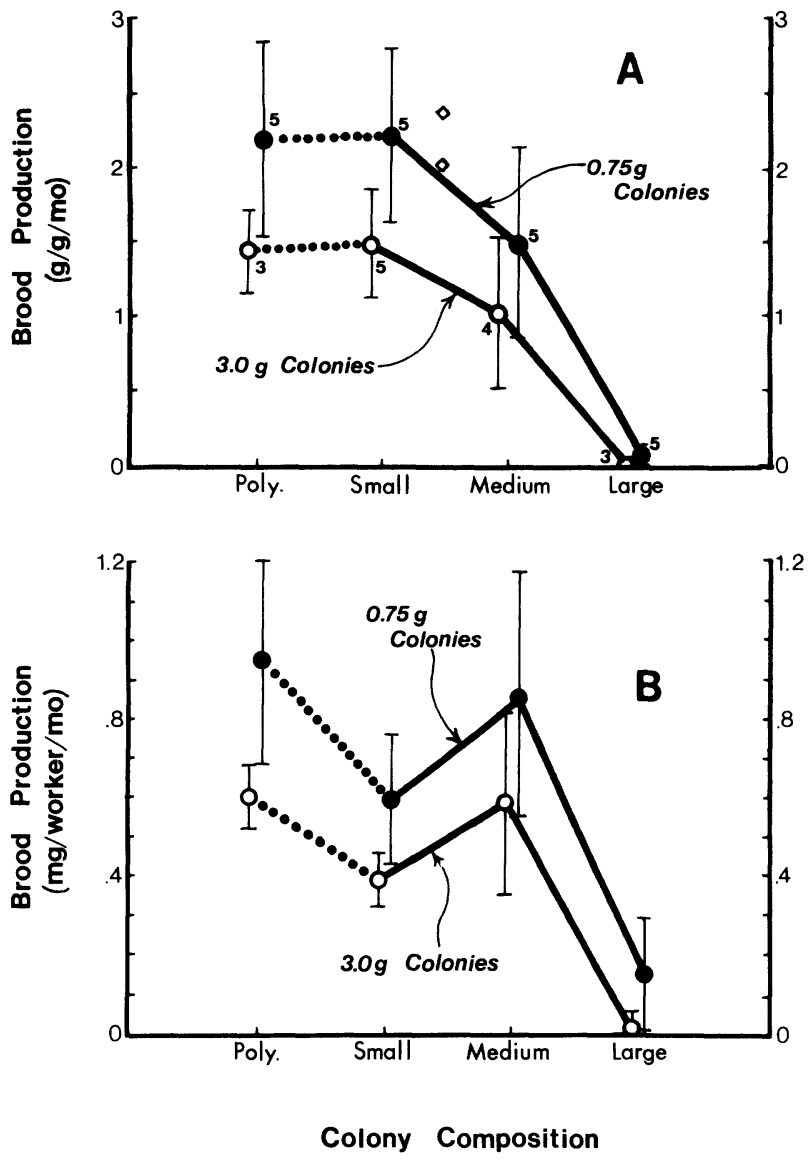

Fig. 2. A Brood production efficiency (dry weight) per gram of workers (dry weight) for polymorphic colonies and experimental monomorphic colonies composed of only small, medium or large workers. Number of observations for each point is shown; bars indicate standard deviations. Open diamonds indicate brood production rates for two $3.0 \mathrm{~g}$ colonies of mediumsmall workers. B Brood production (dry weight) efficiency per worker for polymorphic, small-, medium- and large-worker colonies

BMDP's maximum likelihood analysis (Jennrich and Sampson 1979).

Brood production during the final $2^{1} / 2$ weeks was about twice the rate of the previous 4 weeks, principally because a large portion of the eggs and early instar larvae remained in the colonies after debrooding, but acclimation to laboratory conditions may have also been a factor. Fourth instar larvae began appearing in the colonies after 3-4 days; consequently, the queen's oviposition rate probably remained higher and development of young brood may have been more successful than in the first month.

Rates of brood production both per gram of workers (Fig. $2 \mathrm{~A}, \chi^{2}=12.9,1 d f$ ) and per individual worker (Fig. $2 \mathrm{~B}, \chi^{2}=11.1,1 d f$ ) were significantly higher $(P<0.001)$ in the smaller colonies which grew about $50 \%$ faster than the larger colo- 
nies. The size and composition of workers in experimental colonies also significantly affected brood production rates (Fig. $2 \mathrm{~A}, P=0.01, \chi^{2}=9.2,2 d f$ ); furthermore, the pattern of this effect was independent of colony size $\left(P>0.5, \chi^{2}=0.9,2 d f\right)$. Polymorphic colonies produced brood at about the same rate as colonies composed of only small workers. Medium-worker colonies produced about $30 \%$ less brood and large-worker colonies produced little or no brood. Most eggs laid in largeworker colonies gradually disappeared or failed to develop past third instar larvae. Large-worker colonies were eliminated from the statistical analysis because of unequal variance.

Two $3.0 \mathrm{~g}$ colonies of medium-small workers set up with replicates six and seven produced $20 \%$ more brood than corresponding polymorphic and small-worker colonies (Fig. 2A). An estimate of brood production based on two colonies must be tentative; nevertheless, these data suggest that medium-small workers may be more effective in brood rearing than either polymorphic or smallworker colonies. This possibility is interesting because medium-small workers are the most frequent size class in mature fire ant colonies (Fig. 1). Perhaps brood rearing ability was an important factor in establishing worker size-frequency distributions in fire ant colonies.

A substantial amount of mold grew in the nest chambers of most large-worker colonies, especially in stores of food. The mold probably resulted from improper waste disposal or lax cleaning efforts of the large workers. This problem was not observed in the other experimental treatments.

Polymorphic colonies produced brood at a rate significantly greater $(P<0.01$, paired $t$-test $)$ than that predicted by proportionally adding the brood production rates of the artificial monomorphic colonies (Fig. 2A). Production rates for mediumsmall workers (sieve 30) and medium-large workers (sieve 20) were estimated by interpolation.

Higher production rates in polymorphic colonies were particularly evident if production is examined on a per worker basis (Fig. $2 \mathrm{~B}, \chi^{2}=9.5$, $2 d f$ ). Polymorphic colonies produced significantly more brood per worker $(P<0.01)$ than smallworker colonies and about the same amount as medium-worker colonies. Production rates in large-worker colonies were very low even on a per worker basis. Medium workers appear to be the most skillful worker size class, but polymorphic worker mixtures are equally skillful. These results, however, still need to be verified with experiments in which worker number rather than biomass is held constant. Again brood production rates ac- ross colony composition did not interact significantly $\left(P>0.7, \chi^{2}=0.7,2 d f\right)$ with colony size.

In addition to analyzing statistically the total weight of brood produced in the experimental colonies, we also statistically analyzed the number of brood produced in these colonies and the weight of larvae alone without pupae. Results of these analyses were very similar to those already described for total brood production (Fig. 2A).

Queen weights at the conclusion of the second sample period were significantly lower in the largeworker colonies probably because of the lack of larvae (Tschinkel, unpublished data). Queen weights in polymorphic, small- and mediumworker colonies were not significantly different, although brood production was significantly different.

Worker mortality in polymorphic, small-, medium- and large-worker colonies after six weeks averaged $14 \pm 6 \%, \quad 12 \pm 4 \%, \quad 17 \pm 12 \%$ and $36 \pm 20 \%$ respectively. Mortality rates in largeworker colonies were significantly higher than mortality rates in the other three treatments $(P<$ 0.01 , Duncan's test); other pairwise comparisons were not significantly different. However, large worker mortality in polymorphic colonies averaged $17 \pm 14 \%$, or about half the mortality rate of large workers in artificial monomorphic colonies $\left(P<0.03, F_{1,5}=9.67\right)$. The presence of small workers and/or brood apparently enhances large worker longevity. Worker mortality was also independent of experimental colony size, but highly dependent on source colony $\left(P<0.002, F_{5,24}=\right.$ 5.46). Mortality rates of workers by source colony ranged from $11 \pm 5 \%$ to $32 \pm 23 \%$ and were not associated with rates of brood production.

Curiously, mortality rates of workers in polymorphic, small- and medium-worker colonies were much less than would have been predicted from our estimates of average worker longevity (Fig. 6). This discrepancy may indicate a problem in our estimates of worker longevity, or it may indicate that workers collected from field colonies were much younger than would be expected from a population in equilibrium. Younger than expected age distributions could result from collecting procedures that unintentionally excluded older workers, a rapidly growing population, or field mortality rates that were much higher than those observed in the laboratory. All of the above are likely possibilities.

\section{Egg laying}

The next three experiments were designed to determine at what stage(s) in brood rearing the effects 

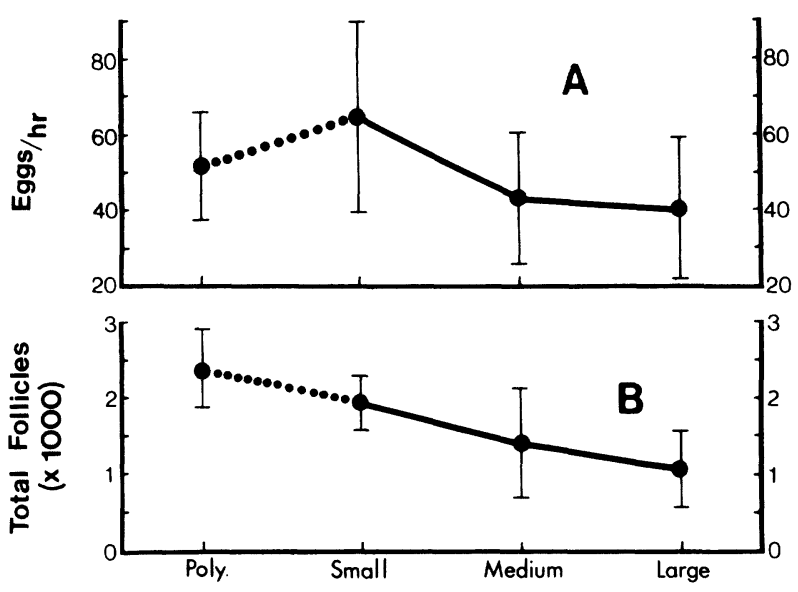

Colony Composition

Fig. 3A, B. Egg laying experiment. A Egg laying rate of queens in polymorphic, small-, medium- and large-worker colonies after 4 days. B Total vitellogenic follicles found in sacrificed queens

observed in the main experiment occurred. The egg laying experiment was conducted to determine if the size and composition of workers in a colony affected the queen's oviposition rate. Four experimental colonies containing either polymorphic, small, medium or large workers were set up from each of six worker source colonies. Each colony contained $1.5 \mathrm{~g}$ of workers and $1.0 \mathrm{~g}$ of larvae pooled from 15-20 laboratory stock colonies. A queen from a laboratory stock colony was randomly adopted into each colony as previously described. Four days after adoption, the egg laying rate of each queen was assessed by direct observation. Following observation, the queens were preserved for dissection and analysis of ovarian development.

The size and composition of workers in experimental colonies did not significantly $(P>0.09)$ affect their adopted queen's egg laying rate (Fig. 3A); nevertheless, the pattern across colony composition was similar to that in the main experiment (Fig. 2A). Colony composition, however, did significantly affect $\left(F_{3,15}=7.08, P=0.004\right)$ the total number of vitellogenic follicles in the queen's ovaries (Fig. 3 B). Total follicles in queens from polymorphic colonies were significantly higher than in queens from medium- and large-worker colonies $(P<0.05$, Duncan's test $)$, and follicles in queens from small-worker colonies were significantly higher than in queens from large-worker colonies. Other combinations were not significantly different. Total vitellogenic follicles is probably a more reliable estimate of a queen's egg laying rate than direct $1 / 2 \mathrm{~h}$ observations because total follicles reflect oviposition rates over a longer time course (Tschinkel, unpublished data) and therefore should not be as variable as short-term observations of egg laying.

\section{Egg rearing}

This experiment investigated the relative egg rearing capabilities of different sized workers. Five worker source colonies were collected from the field 1-2 days prior to use; each was divided into a replicate set of $1.5 \mathrm{~g}$ queenless polymorphic, small-, medium- and large-worker colonies. Experimental colonies were placed in covered $90 \mathrm{~mm}$ plastic petri dishes with a plaster (Castone) floor which covered three-quarters of the dish. The plaster was moistened every 2 days by squirting water through a hole in the cover. The nest was placed in a 22 by $11 \mathrm{~cm}$ plastic box which served as a foraging arena. Each colony in the first three replicate sets received $50 \mathrm{mg}$ of eggs collected from 7-8 day old founding queens; colonies in the remaining two replicate sets received $50 \mathrm{mg}$ of eggs and first instar larvae collected from mature laboratory colonies. The experiment was terminated just prior to the eclosure of the first callow workers; all brood were then counted and weighed.

Founding-queen eggs were larger than eggs from mature queens and included about $50 \%$ trophic eggs (Voss, personal communication). To adjust for these differences, survivorship was calculated as the percent of viable eggs surviving to pupae, and production was calculated as the weight of pupae produced per viable egg. Worker size and composition strongly affected the survivorship of eggs introduced into experimental colonies (Fig. 4A, $F_{3,12}=7.36, P<0.005$ ) and the total weight of pupae produced from them (Fig. 4B, $F_{3,12}=7.09, P<0.006$ ). (Survivorship data were squareroot-arcsine transformed and production data were $\log$ transformed to stabilize variance.) Both survivorship and production declined as the size of workers in monomorphic colonies increased; polymorphic colonies were intermediate between small- and medium-worker colonies. Survivorship of eggs in small-worker colonies was significantly higher than in medium- and largeworker colonies (Duncan's test, $P<0.05$ ). Survivorship in small-worker colonies was significantly higher than in large-worker colonies. Other pairwise comparisons were not significantly different. Production was significantly lower in large-worker colonies $(P<0.05$, Duncan's test $)$ than in the other three treatments. Other pairwise comparisons were 

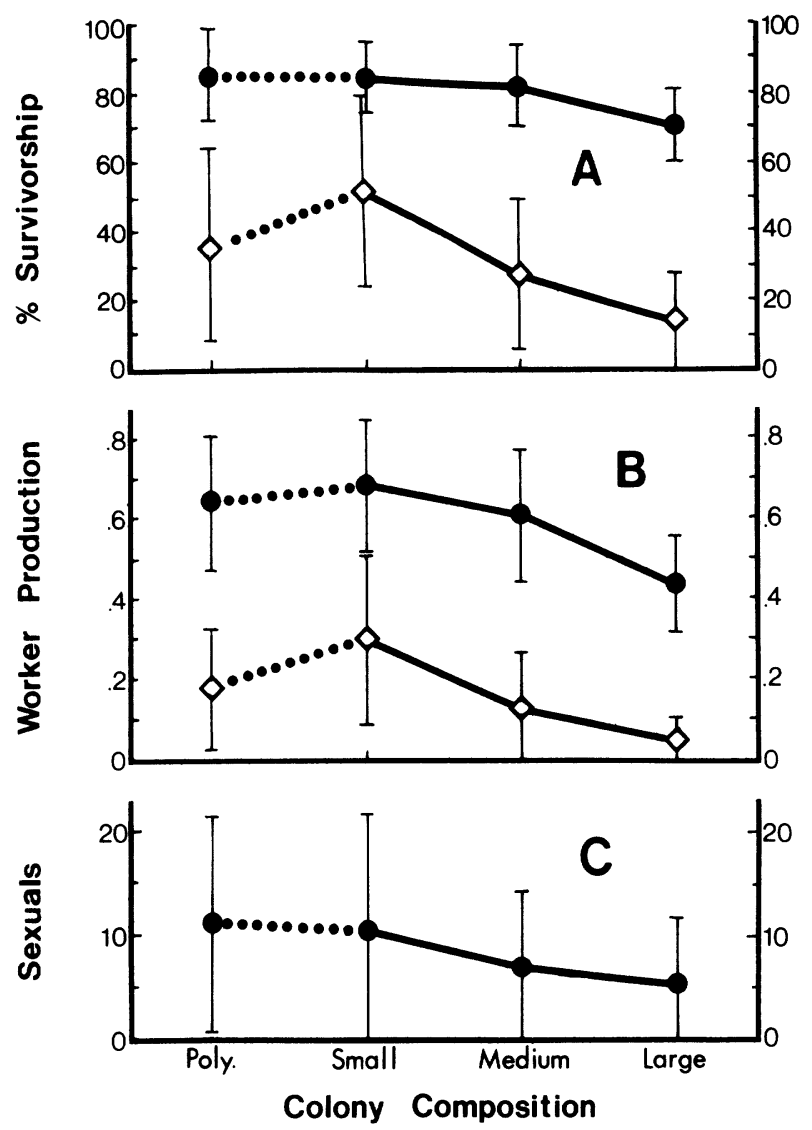

Fig. 4 A-C. Egg and larval rearing experiments. A Percent survivorship of larvae (circles) and viable eggs (diamonds) in colonies of polymorphic, small, medium and large workers. B Production of worker pupae in grams (wet weight) per 1000 larvae (circles) or grams per 1000 viable eggs (diamonds). C Number of sexual brood produced per gram of larvae added. Bars indicate standard deviations

not significantly different. Experimental colonies did not produce sexual brood.

\section{Larval rearing}

This experiment compared larval rearing capabilities of polymorphic, small-, medium- and largeworker colonies. Eight $1.5 \mathrm{~g}$ queenless colonies were formed from each of eight worker source colonies so that sixteen colonies were in each of the four colony composition treatments. Nest design was the same as the egg rearing experiment. Medium sized larvae (mostly early fourth instar, Petralia and Vinson 1979) were collected and pooled from 15-20 laboratory and field colonies for each set of two worker source colonies. One gram of larvae was then added to half of the colonies $(40 \%$ by weight) and $0.25 \mathrm{~g}$ to the other half $(14 \%)$. These levels of brood density were chosen to approximate high and moderate rates of production found in field colonies (Markin et al. 1974). Eleven days after set up, all pupae and the few remaining larvae were collected, counted and weighed.

Larvae added to large-worker colonies pupated 1-2 days before larvae in the other three treatments, perhaps as a result of insufficient feeding although large workers did not overtly neglect their larvae. Survivorship of larvae (Fig. 4A, data squareroot-arcsine transformed, $F_{3,9}=13.18, P<$ 0.002 ) and the total weight of pupae produced from them (Fig. 4B, $F_{3,9}=19.48, P<0.001$ ) was significantly lower in the large-worker colonies than in the other three treatments $(P<0.05$, Duncan's test). Although pairwise comparisons of survivorship and production among polymorphic, small-worker and medium-worker colonies were not significantly different, the pattern across colony composition was very similar to the results of the main experiment (Fig. 2A) and the egg rearing experiment (Fig. 4A and B).

Survivorship and production rates of the high (40\% of total colony weight) and low (14\%) larval densities were not significantly different nor was there a significant interaction between colony composition and larval density. The capacity to produce worker and sexual brood in this experiment was positively associated. The number of sexual brood produced was significantly higher $(P<0.05$, Duncan's test) in polymorphic and small-worker colonies than in medium- and large-worker colonies (Fig. 4C; log transformed data, $F_{3,9}=6.18$, $P<0.015)$. The presence of sexual brood did not appear to reduce the survivorship or production of worker brood.

\section{Respiration}

Four source colonies were collected in October 1982; each colony was sieved into six size classes. Respiration of each size class and of a polymorphic mixture was measured for each colony at two and again at three days after collection. We used $0.5 \mathrm{~g}$ samples rather than individual ants on the assumption that activity rates of ants in groups more nearly approximates activity rates of ants in intact colonies. Secondly, this choice allowed us to obtain an average respiration rate across worker age. Preliminary tests indicated that worker respiration rate was independent of group size $(0.2-0.6 \mathrm{~g})$ and the presence or absence of larvae. Oxygen consumption of experimental samples was measured hourly for $3 \mathrm{~h}$ beginning $1 \mathrm{~h}$ after placing ants into the respiration chambers. Following each respiration run, the ants were killed and dried at $60^{\circ} \mathrm{C}$ for $24 \mathrm{~h}$; they were then counted and weighed. 


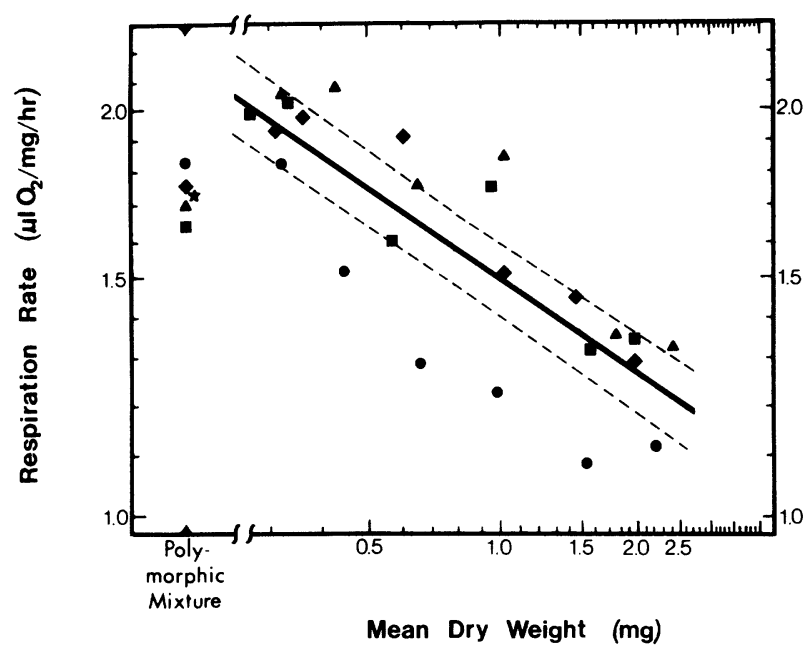

Fig. 5. Worker respiration rates at $30^{\circ} \mathrm{C}$ on day 2 graphed against mean worker dry weight (log-log scale). Data are from four colonies; dashed lines indicate standard error of prediction after adjusting for intercolony differences; $y=1.49 x^{-\mathbf{0 . 2 3 0}}$, $R^{2}=89.2$. Star compares the estimated respiration for polymorphic colonies (calculated from the regression line) with rates actually observed

Workers from different field colonies had significantly different respiration rates; in fact, source colonies accounted for $25 \%$ of data variation (Fig. 5). The number of days after collection was also important because respiration rates declined about $10 \%$ from day 2 to day 3 . The cause of this difference is unknown but possibilities include various stresses, temperature adaptation and dietary changes. We selected data collected on day 2 (Fig. 5) to estimate worker maintenance costs on the assumption that they more accurately reflected respiration rates in field colonies.

Respiration per worker increased almost fivefold from small to large workers $\left(y=1.51 x^{0.805}\right.$, $R^{2}=98 \%$ ), but respiration rates per milligram dry weight (Fig. 8) decreased almost $40 \%$ as worker size increased $\left(y=1.49 x^{-0.230}, R^{2}=89 \%\right.$ after adjusting for intercolony variation). These rates were slightly lower than those reported for other myrmicine ants (Peakin and Josens 1978; Jensen $1978 \mathrm{~b}$ ), but still well within the range of normal variation. The respiration rate of polymorphic worker mixtures equalled that predicted by proportionally summing respiration rates across size classes (Fig. 5). In other words, mixing workers of different sizes did not change their respiration rates.

\section{Longevity}

Callow workers from five field colonies were sorted into 3-5 distinct size classes and marked with wire rings. Short term mortality ( $2 \mathrm{wk}$ ) caused by han-

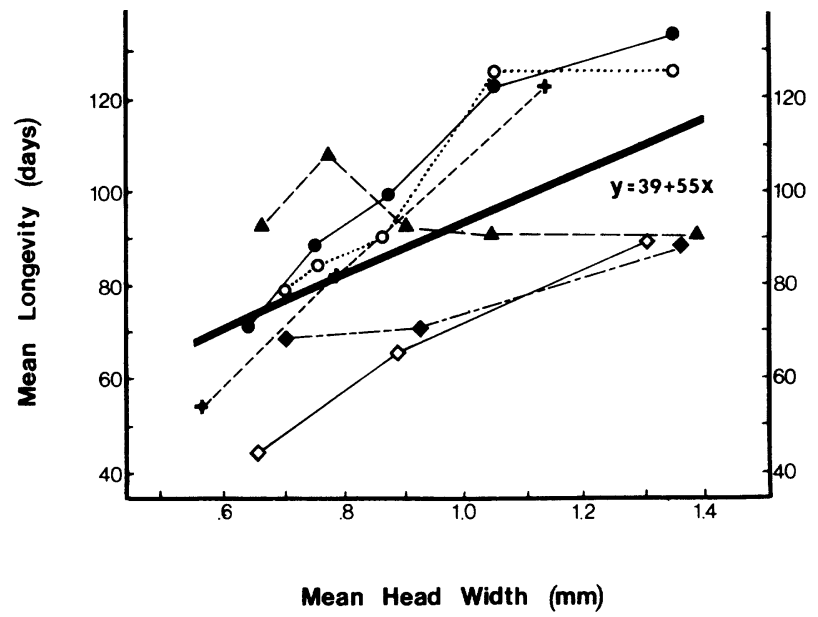

Fig. 6. Mean longevity of fire ant workers at $30^{\circ} \mathrm{C}$ graphed against head width. Each point is the mean of 10-20 workers; $R^{2}=61.2$ after adjusting for intercolony differences. Data are from five colonies and crosses are from Mirenda and Vinson (1981)

dling procedures was less than $10 \%$; workers dying during this period were eliminated from the analysis. Dead workers were collected weekly and checked for wire rings under a dissecting microscope; $92 \%$ of marked ants were eventually recovered.

Worker longevity was a function of body size (Fig. 6). Large workers lived about 50\% longer than small workers. As in the preceeding investigations, intercolony differences strongly affected worker longevity. Longevity among different species of ants also appears size related because largersized species generally live longer than the smaller ones (Peacock and Baxter 1950; Brian 1972; Nielsen 1972; Haskins and Haskins 1980).

\section{Calorimetry}

Caloric content of fire ant tissue varied considerably among colonies; about $60 \%$ of sample variation resulted from intercolony differences. Worker size accounted for an additional $35 \%$ of sample variation (Fig. 7). Caloric content of fire ant workers was $15-25 \%$ higher than values reported for other ants (Rogers et al. 1972; Nielsen 1972; Jensen 1978 a). Caloric content of large worker tissue was $9 \%$ higher than that of small workers $(P<$ $0.001)$. This difference is attributable to higher fat or oil content in large workers. Pellets formed from large workers were much more oily than those from small workers; in fact, great care was necessary to avoid losing this oil when compressing large worker pellets in preparation for burning. Ricks 


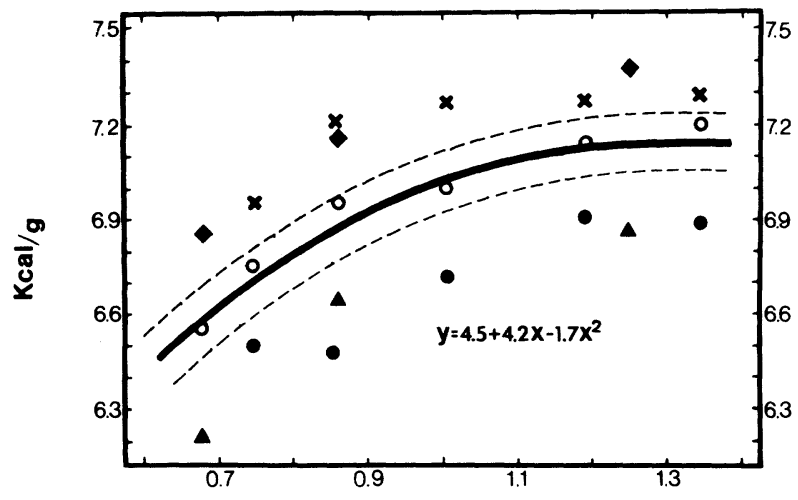

Mean Head Width (mm)

Fig. 7. Relationship between worker size and caloric content (kcal/g dry weight) of workers from five fire ant colonies. Dashed lines indicate standard error of prediction after adjusting for intercolony differences; $R^{2}=95.1$

and Vinson (1972) reported that seasonal fat content of $S$. invicta major workers was consistently higher than that of minor workers; however, this result was not statistically significant, perhaps because they used only two size categories and did not statistically block by source colony. If extra fat or oil reserves can be mobilized for general colony use, major workers may function as a trophic or energy storage caste (Glancey et al. 1973; Howard and Tschinkel 1981); however, this tendency certainly is not strongly developed (Wilson 1978; Mirenda and Vinson 1981).

\section{Discussion}

\section{Worker polymorphism}

Greenberg et al. (in press) reported worker size distributions for monogynous colonies similar to Fig. 1 except that average worker size was slightly larger. They also reported considerable variation in worker size between colonies. Only $5 \%$ of workers in Wilson's (1978) small laboratory colonies had head widths greater than $0.7 \mathrm{~mm}$ compared with $65 \%$ of workers in mature field colonies (Fig. 1 A). This condition is typical of laboratory colonies. Similarly, workers in the polygynous colonies studied by Mirenda and Vinson (1981) were much smaller than workers found in mature monogynous colonies. On the other hand, $58 \%$ of workers in two very large colonies collected by Wood and Tschinkel (1981) had head widths greater than $0.8 \mathrm{~mm}$ as compared to only $24 \%$ in Fig. 1 A. Clearly, the degree of polymorphism differs substantially among colonies. It would be interesting to know why this variation occurs and if colony behavior and activity rates vary correspondingly (Porter and Tschinkel 1985).

$S$. invicta workers display weak allometric growth (Wilson 1953) but strong size variation (Fig. 1). Wilson (1978) termed this condition "weak polymorphism" based on a definition that essentially synonymizes polymorphism with allometry (Wilson 1953). Strong allometry or shape variation is certainly sufficient grounds for polymorphism, but strong size variation should be equally sufficient; after all, labor division in most hymenopteran physical castes is at least as much a function of size variation as it is of shape variation (Wilson 1978; Wilson 1980). We use the term polymorphism in a general sense to describe colony members that are distinctly different in either size or shape. Strong isometric size variation should not be excluded from the term polymorphism (Wilson et al. 1971). S. invicta workers are probably better considered distinctly polymorphic but weakly allometric.

\section{Colony effects}

Intercolony differences accounted for a large proportion of variation in the preceding investigations. Workers from two source colonies in the main experiment failed to produce brood entirely, while workers from the remaining five source colonies produced brood at distinctly different rates. Source colony effects were also evident in the egg laying, egg rearing and larval rearing experiments. Intercolony differences accunted for $25 \%$ of variation in respiration rates (Fig. 5) and $60 \%$ of variation in caloric content (Fig. 7). Similarly, average worker longevity differed substantially between colonies (Fig. 6). Standardized laboratory colonies also show distinctly different food preferences (Glunn et al. 1981).

While differences among field colonies are easily appreciated, differences among standardized laboratory colonies are more difficult to explain. Factors such as worker age, colony health, food preferences and brood production rates may act individually or in concert. Ultimate causes may be genetic, developmental or environmental. Since strong intercolony differences are common, results based on one or a few source colonies should be interpreted carefully and with some skepticism. These differences also suggest that crossing source colonies with experimental treatments may increase statistical sensitivity in the same manner as a paired $t$-test. 


\section{Brood production}

\section{Biomass efficiency}

Brood production efficiency calculated on a biomass basis was highly dependent on colony size (Fig. 2A). Small colonies grew about $50 \%$ faster per gram than the larger colonies, possibly because smaller groups of workers were more efficient at rearing brood or perhaps because queens laid relatively more eggs in the smaller colonies than they did in the larger ones. A number of studies with other social insects indicate that brood production generally declines with increasing colony size (Brian 1953; Michener 1964). There are several empirical reasons why brood production rates might decline as the size of a colony increases. First is the problem of spatial dispersion. Larger colonies require longer lines for communication and resource distribution. Longer lines of distribution, for instance, could mean ineffective or delayed food allocation. Additionally, under field conditions large colonies would require larger foraging areas and longer foraging trips (Jorgensen and Porter 1982), thus decreasing the efficiency of food gathering.

The second problem is one of spatial aggregation. Brian (1956) demonstrated that clumping of brood accounted for decreased worker efficiency in Myrmica rubra because increased worker populations quickly saturated the limited working space available on the brood pile. Care of the queen may be an analogous situation in which an increasing worker population could saturate the space available for her care. Mass recruitment to stationary food sources may be another example where inefficiency is caused by spatial aggregation of workers (Wilson 1962).

A third reason for decreased brood production might be that large colonies shunt a larger percentage of workers from brood care into a reserve population (Mirenda and Vinson 1981). Increased numbers of reserve workers could be adaptive in several ways: (1) They could provide for a more effective colony defense. (2) They could be important in colony survival during the winter or periods of drought. (3) They could act as a hedge against unusual mortality caused by predation or inclement weather. (4) They might also accumulate in preparation for seasonal production of sexual brood.

The size of workers in experimental colonies also strongly affected brood production efficiencies (Fig. 2A). Brood production in the artificial monomorphic colonies decreased as worker size in- creased (Fig. 2A). Larger workers were probably less capable of rearing brood because they are behaviorally less attentive (Wilson 1978) and spend a smaller portion of their life tending brood (Mirenda and Vinson 1981). Additionally, colonies with larger workers have fewer workers per gram; consequently, some tasks may have gone unfilfilled if there were more tasks than workers to fulfill them. Poor sanitary habits of the large workers may have also contributed to their low brood rearing capability.

Brood production in polymorphic and smallworker colonies was nearly equal (Fig. 2A). In other words, polymorphism was neither adaptive nor unadaptive in reference to brood production when food supplies were not limiting.

Polymorphic colonies produced $50 \%$ more brood than predicted by proportionally adding the brood production rates of artificial monomorphic colonies (Fig. 2A, rates for medium-small and medium-large worker colonies were interpolated). The fact that these rates were higher than expected rates may be partially due to interpolation. Two colonies $(3.0 \mathrm{~g})$ of medium-small workers produced $40 \%$ more brood than predicted by interpolation (Fig. 2A). In other words, the brood production curve for artificial monomorphic colonies may actually be an inverted J-shape (Fig. 2), increasing from small- to medium-small-worker colonies and then declining to medium- and largeworker colonies.

However, production rates for polymorphic colonies were still significantly greater than expected even after using $40 \%$ higher production rates for medium-small workers. A second possibility for this effect is synergism. Different sized workers may be more efficient when they work together in polymorphic colonies than when they work separately in monomorphic colonies. Large workers, for instance, may be almost unable to rear brood in colonies by themselves because they do not perform several critical functions normally performed by small workers. If so, large workers in polymorphic colonies are probably capable of contributing substantially more to brood rearing than large workers in monomorphic colonies. Large workers might also increase brood production if they perform functions in polymorphic colonies not ordinarily performed by small workers.

The degree of polymorphism in field colonies increases with their size and age (Markin et al. 1973). This relationship suggests that the relative benefits of polymorphism may also increase with colony size. Our experiments, however, do not support this hypothesis since the pattern of brood pro- 
duction among polymorphic, small-, medium- and large-worker colonies was the same at both levels of colony size (Fig, 2A); in short, the relative advantage of polymorphism did not increase with colony size. It should be noted, however, that we investigated only brood production and our experimental colonies were considerabely smaller than mature field colonies. Conceivably, very large polymorphic colonies could still produce more brood than very large colonies of small workers.

The results of the egg laying, egg rearing and larval rearing experiments indicated that the size of workers in a colony affected brood rearing success during all stages of development (Figs. 3 and 4). Worker size affected queen oviposition rate, the survivorship of young larvae and the amount of pupae produced. Furthermore, the pattern of results observed in these experiments agreed with those in the main experiment (Fig. 2A) in that brood production capabilities in the artificial monomorphic colonies consistantly declined as worker size increased and also because polymorphic and small-worker colonies were not significantly different in brood rearing capabilities. The egg rearing experiment (Fig. 4) indicated that low survivorship of eggs and small larvae may be a major cause of lowered brood production in colonies with larger workers. Feedback from late fourth instar larvae stimulates queen oviposition (Tschinkel, unpublished data) and perhaps also the development of small larvae. Consequently, an initially low production of larvae may reduce brood production in subsequent generations as well.

\section{Energetic efficiency}

On an energetic basis, brood production efficiency (Fig. 9) is the quantity of brood produced per unit time (Fig. 2A) divided by the energy expended in production and maintenance (Fig. 8). Maintenance costs were estimated by measuring worker respiration rates (Fig. 5). Worker production costs include larval respiration and the energy incorporated into worker tissue (Fig. 7).

Respiration costs for larvae which developed into small workers were about $15 \%$ of total energy costs for producing small workers (unpublished data). Unfortunately, we did not determine respiration costs for the larvae of other worker size classes because of difficulty in determining both the respiration rates and the duration of each developmental stage for each worker size class. Nevertheless, even rather strong differences in larval respiration between worker size classes would not affect total costs (Fig. 8) by more than a few per-

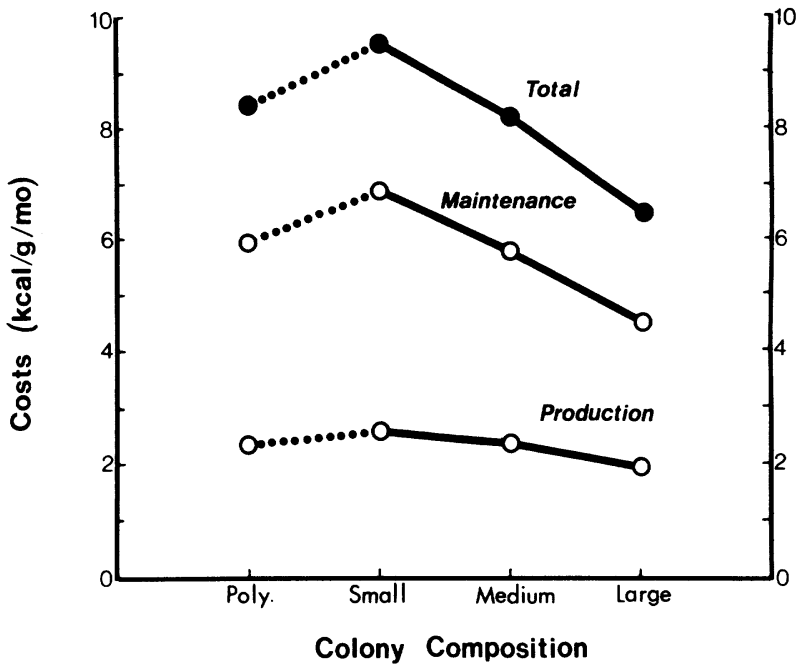

Fig. 8. Estimated costs of producing (exclusive of larval respiration) and maintaining one gram of polymorphic, small, medium or large workers for 1 month

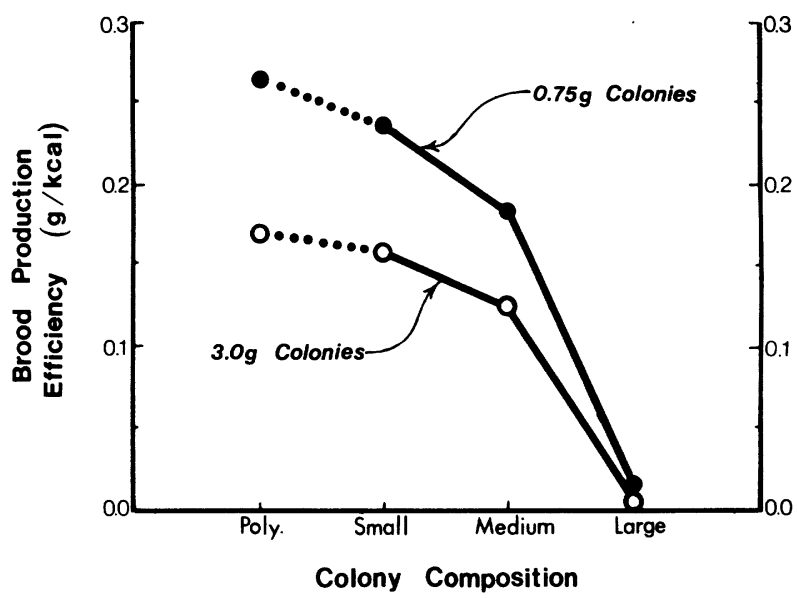

Fig. 9. Energetic brood production efficiencies of polymorphic, small-, medium- and large-worker colonies in grams of brood produced per kcal of energy expended

cent because larval respiration costs are relatively low and are primarily a function of worker longevity.

Our data indicate that it is less costly to produce workers than it is to maintain them. Production costs (less larval respiration) were about $30 \%$ of total costs (Fig. 8). This percentage agrees well with estimates for other species of ants (Jensen 1978 b). Production costs including larval respiration were about $40 \%$ of total energy costs.

Energy costs for one gram of large workers were about $30 \%$ less than for an equal weight of small workers (Fig. 8), primarily because large workers lived longer (Fig. 6) and had lower metabolic rates (Fig. 5). Similarly, energy costs for one 
gram of polymorphic workers were about $10 \%$ less than for an equivalent weight of small workers.

A word of caution is necessary because our estimates of brood production and energetic costs were determined in laboratory colonies at $30^{\circ} \mathrm{C}$. Brood production and energy costs experienced under field conditions may be quite different than those measured in the laboratory (Nielsen and Jensen 1977); however, even fairly major differences would not be too critical as long as they did not interact with worker size.

Energetic efficiencies were highly dependent on colony size (Fig. 9). Small colonies $(0.75 \mathrm{~g})$ were $50 \%$ more efficient than the larger colonies $(3.0 \mathrm{~g})$. The energetic efficiency of polymorphic colonies was about $10 \%$ higher than it was for small-worker colonies. As worker size increased in the artificial monomorphic colonies, efficiencies decreased (Fig. 9). Small workers or perhaps medium-small workers appear to be the optimal brood rearing size class (Fig. 2A).

\section{Conclusion}

Polymorphic and small-worker colonies of equal weight produced brood at equivalent rates (Fig. 2A). In other words when food supplies are not limiting, polymorphism is apparently neither an adaptive advantage nor a disadvantage. However, polymorphic colonies are energetically more efficient (Fig. 9), and under food-limited conditions, polymorphism may offer a small adaptive advantage in brood production depending on the degree of food stress. This prediction, however, needs to be tested experimentally. Future ergonomic studies of foraging, defense, sexual production and nest maintenance are still required before the overall adaptive value of fire ant polymorphism can be ascertained.

Acknowledgements. I wish to thank my wife, Colleen K. Porter, for her support and technical assistance in preparing this manuscript; she has been patient and understanding throughout my graduate career. A traineeship provided by the Psychobiology Research Program at Florida State University greatly facilitated this research.

\section{References}

Banks WA, Lofgren CS, Jouvenaz DP, Stringer CE, Bishop PM, Williams DF, Wojcik DP, Glancey BM (1981) Techniques for collecting, rearing, and handling imported fire ants. USDA and SEA, Adv Agricult Technol Southern Ser 21:1-9

Brian MV (1953) Brood-rearing in relation to worker number in the ant Myrmica. Physiol Zool 26:355-366

Brian MV (1956) Group form and causes of working inefficiency in the ant Myrmica rubra L. Physiol Zool 29:173-194
Brian MV (1972) Population turnover in wild colonies of the ant Myrmica. Ekol Polska 20:43-53

Davies SP (1966) A constant pressure respirometer for mediumsized animals. Oikos 17:108-112

Ettershank G, Whitford WG (1973) Oxygen consumption of two species of Pogonomyrmex harvester ants (Hymenoptera: Formicidae). Comp Biochem Physiol 46 [A]:605-611

Elliott JM, Davison W (1975) Energy equivalents of oxygen consumption in animal energetics. Oecologia (Berl) 19:195-201

Glancey BM, Stringer CE, Craig CH, Bishop PM, Martin BB (1973) Evidence of a replete caste in the fire ant Solenopsis invicta. Ann Entomol Soc Am 66:233-234

Glunn FJ, Howard DF, Tschinkel WR (1981) Food preference in colonies of the fire ant Solenopsis invicta. Insectes Soc 28:217-222

Greenberg L, Fletcher DJC, Vinson SB (in press) Differences in worker size and mound distribution in monogynous and polygynous colonies of the fire ant Solenopsis invicta Buren. J Kansas Entomol Soc

Haskins CP, Haskins EF (1980) Notes on female and worker survivorship in the archaic ant genus Myrmecia. Insectes Soc 27:345-350

Hays SB, Horton PM, Bass JA, Stanley D (1982) Colony movement of imported fire ants. J Ga Entomol Soc 17:266-274

Howard DF, Tschinkel WR (1981) Internal distribution of liquid foods in isolated workers of the fire ant, Solenopsis invicta. J Insect Physiol 27:67-74

Jennrich R, Sampson P (1979) General mixed model analysis of variance. In: Dixon WJ, Brown MB (eds) BMDP biomedical computer programs, P-series. University of California Press, Berkeley, pp 581-598

Jensen TF (1978a) An energy budget for a field population of Formica pratensis Retz. (Hymenoptera: Formicidae). Natura Jutl 20:203-226

Jensen TF (1978b) Annual production and respiration in ant populations. Oikos 31:207-213

Jorgensen CD, Porter SD (1982) Foraging behavior of Pogonomyrmex owyheei in southeast Idaho. Environ Entomol $11: 381-384$

Markin GP, Dillier JH, Collins HL (1973) Growth and development of colonies of the red imported fire ant, Solenopsis invicta. Ann Entomol Soc Am 66:803-808

Markin GP, O’Neal J, Dillier JH, Collins HL (1974) Regional variation in the seasonal activity of the imported fire ant, Solenopsis saevissima richteri. Environ Entomol 3:446452

Michener CD (1964) Reproductive efficiency in relation to colony size in hymenopterous societies. Insectes Soc $11: 317-341$

Mirenda JT, Vinson SB (1979) A marking technique for adults of the red imported fire ant (Hymenoptera:Formicidae). Fla Entomol 62:279-281

Mirenda JT, Vinson SB (1981) Division of labour and specification of castes in the red imported fire ant Solenopsis invicta Buren. Anim Behav 29:410-420

Nielsen MG (1972) An attempt to estimate energy flow through a population of workers of Lasius alienus (Först) (Hymenoptera:Formicidae). Natura Jutl 16:99-107

Nielsen MG, Jensen TB (1977) Measurement of metabolism of worker ants by using the elimination of caesium-134. Oecologia (Berl) 30:209-214

O’Neal J, Markin GP (1973) Brood nutrition and parental relationships of the imported fire ant Solenopsis invicta. J Ga Entomol Soc 8:294-303

Oster GF, Wilson EO (1978) Caste and ecology in the social insects. Princeton University Press, Princeton 
Peacock AD, Baxter AT (1950) Studies in Pharaoh's ant, Monomorium pharaonis (L.). 3. Life history. Entomol Mon Mag 86:171-178

Peakin GJ, Josens G (1978) Respiration and energy flow. In: Brian MV (ed) Production ecology of ants and termites. IBP synthesis, vol 13. Cambridge University Press, Cambridge, pp 111-163

Petralia RS, Vinson SB (1978) Feeding in the larvae of the imported fire ant, Solenopsis invicta: behavior and morphological adaptations. Ann Entomol Soc Am 71:643-648

Petralia RS, Vinson SB (1979) Developmental morphology of larvae and eggs of the imported fire ant, Solenopsis invicta. Ann Entomol Soc Am 72:472-484

Porter SD (1983) Fast, accurate method of measuring ant head widths. Ann Entomol Soc Am 76:866-867

Porter SD, Jorgensen CD (1981) Foragers of the harvester ant, Pogonomyrmex owyheei: A disposable caste? Behav Ecol Sociobiol 9:247-256

Porter SD, Tschinkel WR (1985) Fire ant polymorphism (Hymenoptera: Formicidae): factors affecting worker size. Ann Entomol Soc Am (in press)

Ricks BL, Vinson SB (1972) Changes in nutrient content during one year in workers of the imported fire ant. Ann Entomol Soc Am 65:135-138

Rogers L, Lavigne R, Miller JL (1972) Bioenergetics of the western harvester ant in the shortgrass plains ecosystem. Environ Entomol 1:763-768
Shoemaker DP, Garland CW, Steinfeld JI, Nibler JW (1981) Experiments in physical chemistry, 4th edn. McGraw-Hill, New York

Tschinkel WR, Howard DF (1978) Queen replacement in orphaned colonies of the fire ant, Solenopsis invicta. Behav Ecol Sociobiol 3:297-310

Wilson EO (1953) The origin and evolution of polymorphism in ants. Q Rev Biol 28:136-156

Wilson EO (1962) Chemical communication among workers of the fire ant Solenopsis saevissima (Fr. Smith) 1. The organization of mass-foraging. Anim Behav 10:134-147

Wilson EO (1968) Ergonomics of caste in the social insects. Am Nat 102:41-66

Wilson EO (1975) Sociobiology: the new synthesis. Belknap Press of Harvard University Press, Cambridge, Mass

Wilson EO (1978) Division of labor in fire ants based on physical castes (Hymenoptera: Formicidae: Solenopsis). J Kansas Entomol Soc $51: 615-636$

Wilson EO (1980) Caste and division of labor in leaf-cutter ants (Hymenopera:Formicidae: Atta). II. The ergonomic optimization of leaf cutting. Behav Ecol Sociobiol 7:157-165

Wilson NL, Dillier JH, Markin GP (1971) Foraging territories of imported fire ants. Ann Entomol Soc Am 64:660-665

Wood LA, Tschinkel WR (1981) Quantification and modification of worker size variation in the fire ant Solenopsis invicta. Insectes Soc 28:117-128 